\title{
Performance Analysis of a Two-Hop MIMO Mobile-to-Mobile via Stratospheric-Relay Link Employing Hierarchical Modulation
}

\author{
Nikolaos Nomikos, ${ }^{1}$ Emmanouel T. Michailidis, ${ }^{2}$ \\ Demosthenes Vouyioukas, ${ }^{1}$ and Athanasios G. Kanatas ${ }^{2}$ \\ ${ }^{1}$ Department of Information and Communication Systems Engineering, University of the Aegean, 83200 Karlovassi, Samos, Greece \\ ${ }^{2}$ Department of Digital Systems, University of Piraeus, 80 Karaoli \& Dimitriou Street, 18534 Piraeus, Greece
}

Correspondence should be addressed to Demosthenes Vouyioukas; dvouyiou@aegean.gr

Received 12 March 2013; Revised 21 May 2013; Accepted 27 May 2013

Academic Editor: Fernando Pérez Fontán

Copyright ( $) 2013$ Nikolaos Nomikos et al. This is an open access article distributed under the Creative Commons Attribution License, which permits unrestricted use, distribution, and reproduction in any medium, provided the original work is properly cited.

\begin{abstract}
Next generation wireless communication networks intend to take advantage of the integration of terrestrial and aerospace infrastructures. Besides, multiple-input multiple-output (MIMO) architecture is the key technology, which has brought the wireless gigabit vision closer to reality. In this direction, high-altitude platforms (HAPs) could act as relay stations in the stratosphere transferring information from an uplink to a downlink MIMO channel. This paper investigates the performance of a novel transmission scheme for the delivery of mobile-to-mobile (M-to-M) services via a stratospheric relay. It is assumed that the source, relay, and destination nodes are equipped with multiple antennas and that amplify-and-forward (AF) relaying is adopted. The performance is analyzed through rigorous simulations in terms of the bit-error rate (BER) by using a recently proposed 3D geometry-based reference model in spatially correlated flat-fading MIMO channels, employing a hierarchical broadcast technique and minimum mean square error (MMSE) receivers.
\end{abstract}

\section{Introduction}

Mobile-to-mobile (M-to-M) communications are expected to be a fundamental component of future mobile ad hoc networks, intelligent highway vehicular systems (IHVS), broadband mobile multimedia services, and relay-based cellular networks. Although the radio links of M-to-M systems are usually dominated by harsh multipath fading effects, applying cooperative diversity techniques by utilizing single or multiple intermediate relays $(R)$ between a source $(S)$ and a destination $(D)$ can preserve the end-to-end communication [1-3]. As the evolution of next-generation networks (NGN) promises the integration of heterogeneous wireless terrestrial and aerospace technologies [4-6], employing quasistationary high altitude platforms (HAPs) [7] as radio-relay nodes in the stratosphere is a potential and attractive solution for the interconnection of existing infrastructures [8-10]. Previous work on relaying through HAPs considered a multitude of scenarios, such as the provision of surveillance, monitoring, maritime, and third-generation (3G) cellular serviceslinebreak [10-14].

To provide appreciable diversity and spatial multiplexing gains over multipath environments, the application of multiple-input multiple-output (MIMO) technology and space-time coding schemes is suggested in current and future wireless standards $[15,16]$. Nevertheless, the successful design and deployment of MIMO M-to-M via stratospheric relay (MMSR) systems require a detailed knowledge of the underlying radio channel and the mechanisms of radio wave propagation. Using this knowledge, communication systems can be constructed to obtain optimal or near optimal performance. Notwithstanding, the MIMO benefits may not be attained in practice due to the channel specificity [17], for example, system geometry, fading type, correlation at the transmit and/or receive antennas, and transceiver impairments. In addition, the adaptation of the modulation at 
the transmitter side according to the channel characteristics allows the reduction of the transmission power and/or the enhancement of the data rates [18]. Besides, adaptive usage of the resources offered by the various modulation and coding schemes is mandatory for a system's efficiency and economy. Therefore, using detectors, the effect of fading and correlation can be overcome [19], while taking advantage of the adaptive techniques, thus leading to adaptive operation of the receiver without any control commands.

In this paper, the performance analysis of a MIMO MMSR system is analyzed through simulations in terms of bit-error-rate (BER) so as to estimate the complementary role of HAPs in conjunction with terrestrial networks and reap the benefits of MIMO MMSR communications. It is well known that spatial multiplexing systems that employ linear receiver schemes, either zero-forcing (ZF) or minimum mean square error (MMSE), are practically important because of their minimal complexity requirements $[20,21]$. This paper focuses on the performance of MMSE detectors at the receivers over spatially correlated double-Rician flat-fading MIMO MMSR channels, since these receivers offer better performance than $\mathrm{ZF}$ receivers and avoid the noise-enhancement effect. It is considered that hierarchically modulated symbols [22] are transmitted in each time slot through the stratospheric relay in order to distinguish receivers and offer upgraded service quality to those with better reception conditions as well as backward compatibility.

Since impairments of the signal are mainly caused by the environment near the user, the starting point of the performance evaluation is a recently proposed three-dimensional (3D) reference geometry-based model for MIMO MMSR channels in dual-hop amplify-and-forward (AF) networks [23]. This model enables a realistic positioning of the scatterers in the vicinity of the source and the destination and assumes that these scatterers are nonuniformly distributed within two cylinders, which reflect the influence of two heterogeneous $3 \mathrm{D}$ nonisotropic scattering environments. The communication between the source and the destination is only feasible through a relay due to high attenuation in the propagation medium. While the utilized carrier frequencies are below $10 \mathrm{GHz}$, both line-of-sight (LoS) and non-lineof-sight (NLoS) propagation conditions may exist in the transmission links from the source to the destination via the stratospheric relay, whereas rain effects are insignificant.

The remainder of this paper is organized as follows. Section 2 outlines the MIMO MMSR system model and the modulation techniques employed. Section 3 presents the MIMO MMSR channel model and analyzes the propagation characteristics. The performance analysis along with the corresponding results is presented in Section 4. Finally, conclusions and future research directions are given in Section 5.

\section{System Model}

The system model employed throughout this chapter considers a narrowband MIMO MMSR fading channel with $L_{S}$ antenna elements at a terrestrial source mobile station, $L_{R}$ antenna elements at a half-duplex stratospheric relay station

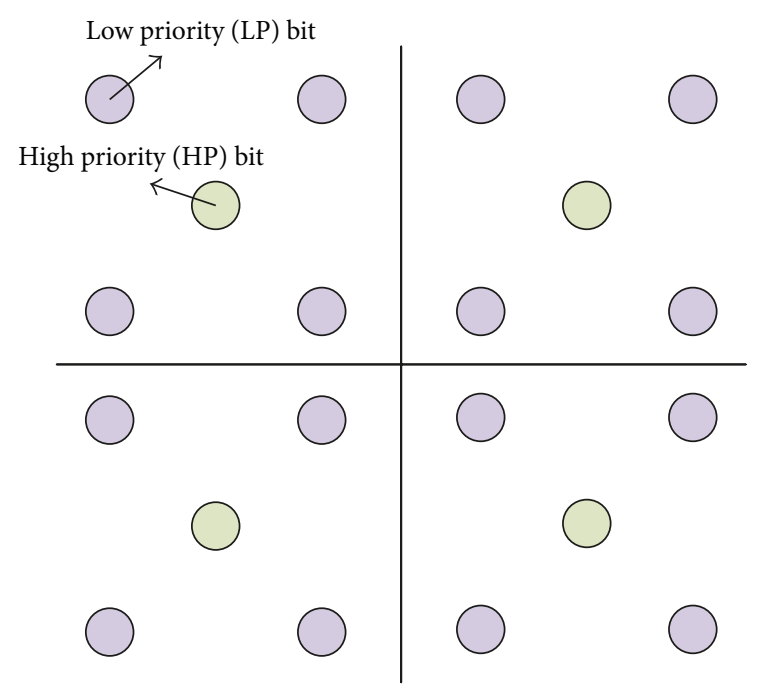

FIGURE 1: Hierarchical modulation of order 4/16QAM.

free of local scattering supporting a two-hop communication, and $L_{D}$ antenna elements at a terrestrial destination mobile station. All antennas are omnidirectional and are numbered as $1 \leq p \leq p^{\prime} \leq L_{S}, 1 \leq q \leq q^{\prime} \leq L_{R}$, and $1 \leq l \leq$ $l^{\prime} \leq L_{D}$, respectively. The source and destination arrays are equipped with low-elevation antennas, while the relay flies at a height $h_{R} \approx 20 \mathrm{~km}$ above the ground. The relatively short endurance due to fuel constraints and human factors limits the deployment of manned HAPs as relay nodes. Therefore, it is assumed that the relay is a solar-powered unmanned quasistationary airship.

Let us consider that the destination receives data from the source only through the relay, since the direct sourcedestination transmission link is totally obstructed. Thus, the communication takes place in two time slots. The relay receives the source signal in each time slot and sends it to the destination through an AF process. In each transmission, the source employs hierarchical modulation with two priority streams, where the high-priority (HP) robust symbols are QPSK modulated, while the low-priority (LP) symbols are 16QAM modulated. The constellation diagram of the 4/16QAM hierarchically modulated symbols is illustrated in Figure 1, where the bits corresponding to each priority stream are distinguished. Each time slot is divided in $N$ symbol periods, where $N$ is the size in symbols of each transmitted source signal vector. These streams are fed to the source and after the two-hop transmission the destination will try to decode either the LP stream, if channel conditions are favorable, or the HP, which is more robust to bad link quality.

Assuming frequency-flat fading channels and constant channel coefficients for duration equal to $N$ symbol periods, that is, one time slot, in the source-relay $(S-R)$ and relaydestination $(R-D)$ links, the signal received at the relay is

$$
\mathbf{y}_{R}=\mathbf{H}_{S R} \mathbf{x}+\mathbf{n}_{R}
$$


where $\mathbf{y}_{R} \in \mathbb{C}^{L_{R} \times N}, \mathbf{H}_{S R} \in \mathbb{C}^{L_{R} \times L_{S}}$ are the complex channel coefficients in the $S$ - $R$ link, $\mathbf{x} \in \mathbb{C}^{L_{S} \times N}$ is the transmitted source signal vector, and $\mathbf{n}_{R} \in \mathbb{C}^{L_{R} \times N} \sim C \mathcal{N}\left(\mathbf{0}, \mathbf{I}_{L_{R}}\right)$ is the additive white Gaussian noise (AWGN) at the relay, with $\mathbf{I}_{L_{R}}$ the identity matrix of size $L_{R} \times L_{R}$. The relay amplifies $\mathbf{y}_{R}$ with a factor $A$; thus, the received signal vector at the destination in the second time slot is

$$
\begin{aligned}
\mathbf{y}_{D} & =\mathbf{H}_{R D} A \mathbf{y}_{R}+\mathbf{n}_{D}=\mathbf{H}_{R D} A\left(\mathbf{H}_{S R} \mathbf{x}+\mathbf{n}_{R}\right)+\mathbf{n}_{D} \\
& =\mathbf{H}_{R D} A \mathbf{H}_{S R} \mathbf{x}+\mathbf{H}_{R D} A \mathbf{n}_{R}+\mathbf{n}_{D},
\end{aligned}
$$

where $\mathbf{y}_{D} \in \mathbb{C}^{L_{D} \times N}, \mathbf{H}_{R D} \in \mathbb{C}^{L_{D} \times L_{R}}$ are the complex channel coefficients in the $R-D$ link and $\mathbf{n}_{D} \in \mathbb{C}^{L_{D} \times N} \sim C \mathcal{N}\left(\mathbf{0}, \mathbf{I}_{L_{D}}\right)$ is the AWGN at the destination, with $\mathbf{I}_{L_{D}}$ the identity matrix of size $L_{D} \times L_{D}$. At the destination, the reception is based on minimum mean square error (MMSE) equalization, which uses the knowledge of the noise variance in order to maximize the signal-to-interference-plus-noise ratio (SINR). Retrieval of the source signal is based on matrix $\mathbf{W}_{\text {MMSE }}$ which is denoted as

$$
\mathbf{W}_{\mathrm{MMSE}}=\left(\mathbf{H}^{H} \mathbf{H}+\sigma_{w}^{2} \mathbf{I}_{L_{S}}\right)^{-1} \mathbf{H}^{H},
$$

where $(\cdot)^{H}$ denotes the Hermitian matrix, $\mathbf{H}=\mathbf{H}_{R D} A \mathbf{H}_{S R}$ is the end-to-end $S-R-D$ channel matrix [24], and $\sigma_{w}^{2}$ is the variance of the total noise $\mathbf{n}_{W}=\mathbf{H}_{R D} A \mathbf{n}_{R}+\mathbf{n}_{D}$ which contains both the amplified version of noise $\mathbf{n}_{R}$ and the noise at the destination $\mathbf{n}_{D}$. The $i$ th row of $\mathbf{W}_{\text {MMSE }}$ is derived by solving the optimization equation

$$
w_{i . \mathrm{MMSE}}=\underset{\substack{w=\left(w_{1}, \ldots, w_{L_{S}}\right) \\ \operatorname{argmax}}}{\left|\mathbf{w h}_{i}\right|^{2} E_{x}} \frac{\sum_{j=1, j \neq i}^{L_{S}}|\mathbf{w h}|^{2}+\|\mathbf{w}\|^{2} \sigma_{w}^{2}}{2}
$$

where $\|\cdot\|$ stands for the Euclidean norm of a vector, $\mathbf{h}_{i}$ is the $i$ th column of the channel matrix $\mathbf{H}$, and $E_{x}$ is the power of the source signal. The output of the MMSE equalizer will be

$$
\widehat{\mathbf{x}}=\mathbf{W}_{\mathrm{MMSE}} \mathbf{y}=\mathbf{x}+\left(\mathbf{H}^{H} \mathbf{H}+\sigma_{w}^{2} \mathbf{I}_{L_{S}}\right)^{-1} \mathbf{H}^{H} \mathbf{n}_{w} .
$$

Since $\mathbf{H}=\mathbf{H}_{R D} A \mathbf{H}_{S R}$, one can first derive the matrices $\mathbf{H}_{S R}$ and $\mathbf{H}_{R D}$ and then derive the matrix $\mathbf{H}$. The $S-R$ MIMO channel is Rician in general. Hence, $\mathbf{H}_{S R}$ can be modeled as [15]

$$
\mathbf{H}_{S R}=\sqrt{\frac{K_{S R}}{K_{S R}+1}} \mathbf{H}_{S R, \mathrm{LoS}}+\sqrt{\frac{1}{K_{S R}+1}} \mathbf{H}_{S R, \mathrm{NLoS}},
$$

where $\mathbf{H}_{S R \text {,LoS }}$ is the matrix containing the free-space (LoS) responses between all source-relay elements, $\mathbf{H}_{S R, N L o S}$ accounts for the scattered (NLoS) signals, and $K_{S R}$ is the Rician factor. The elements of $\mathbf{H}_{S R \text {,LoS }}$ are deterministic and can be directly obtained using the reference model in [23], while the matrix $\mathbf{H}_{S R, N L o S}$ can be evaluated as follows [25]:

$$
\operatorname{vec}\left(\mathbf{H}_{S R, \mathrm{NLoS}}\right)=\mathbf{R}_{S R, \mathrm{NLoS}}^{1 / 2} \operatorname{vec}\left(\mathbf{H}_{w}\right),
$$

where $\mathbf{R}_{S R, N L o S}$ is the $L_{R} L_{S} \times L_{R} L_{S}$ correlation matrix associated with the NLoS component of the $S-R$ MIMO subsystem, $\mathbf{R}_{S R, \mathrm{NLOS}}^{1 / 2}$ is the square root of $\mathbf{R}_{S R, \mathrm{NLoS}}$ that satisfies $\mathbf{R}_{S R, \mathrm{NLOS}}^{1 / 2} \mathbf{R}_{S R, \mathrm{NLOS}}^{H / 2}=\mathbf{R}_{S R, \mathrm{NLOS}}, \mathbf{H}_{w}$ is a $L_{R} \times L_{S}$ stochastic matrix with independent and identically distributed (i.i.d.) zero mean complex Gaussian entries, and vec $(\cdot)$ denotes matrix vectorization (The vectorization of a matrix is a linear transformation which converts the matrix into a column vector. Specifically, the vectorization of a $m \times n$ matrix $A$ denoted by $\operatorname{vec}(A)$ is the $m n \times 1$ column vector obtained by stacking the columns of the matrix $A$ on top of one another.). Since $\mathbf{H}_{w}$ is stochastic, $\mathbf{H}_{S R \text {,NLoS }}$ varies for each simulation trial and converges to the desired one in the statistical sense, that is, when averaged over a sufficiently large number of simulation trials. Note that $\mathbf{H}_{R D}$ can be derived by following a similar procedure to that used to derive $\mathbf{H}_{S R}$.

\section{Propagation Characteristics and Channel Modeling}

In this section, the 3D model for MIMO MMSR channels proposed in [23] is described and the corresponding propagation characteristics are underlined. Based on this model, the MIMO channel matrix of the $S-R-D$ system can be achieved and then the performance investigation of the MIMO MMSR system can be accomplished.

It is considered that the source and the destination are located within two separate cylindrical regions of scattering elements. Specifically, the scatterers in the vicinity of the source and the destination are nonuniformly distributed within two cylinders, which reflect the influence of two heterogeneous $3 \mathrm{D}$ scattering environments. The radius of cylinder 1 (2) corresponds to the maximum distance between the source (destination) and an effective scatterer in the azimuth domain, while the maximum scatterer height corresponds to the height of cylinder 1 (2). The waves emitted from the source (relay) antennas travel over paths of different lengths and impinge the relay (destination) antennas from different directions due to the $3 \mathrm{D}$ non-isotropic scattering conditions within cylinder 1 (2).

The geometrical characteristics of this model are discussed in Figures 2 and 3. Figure 2 presents the LoS paths of the $3 \mathrm{D}$ model for a $2 \times 2 \times 2$ MIMO MMSR channel. One observes that the location of the relay seen from the source (destination) can be specified by the azimuth angle $\omega_{S}\left(\omega_{D}\right)$ and the elevation angle $\beta_{S}\left(\beta_{D}\right)$ relative to the $x y$ plane. The antenna interelement distance at the source, relay, and destination is denoted by $\delta_{S}, \delta_{R}$, and $\delta_{D}$, respectively. In addition, the orientation of the antenna arrays at the source, relay, and destination is described by the angles $\theta_{S}, \theta_{R}$, and $\theta_{D}$, respectively, while $\psi_{S}$ and $\psi_{D}$ denote the elevation angle of the $p$ th source and the $l$ th destination antenna element.

Figure 3 presents the single-bounce NLoS paths for the channel in Figure 2. It is assumed that $M \rightarrow$ $\infty(N \rightarrow \infty)$ scatterers are situated within a cylinder of radius $R_{S \text {, max }}\left(R_{D \text {, max }}\right)$ and height $H_{S \text {, max }}\left(H_{D \text {, max }}\right)$ around the source (destination). Let $S_{S}^{(m)}\left(S_{D}^{(n)}\right)$ be the $m$ th $(n$ th) scatterer in the vicinity of the source (destination). The radial distance 


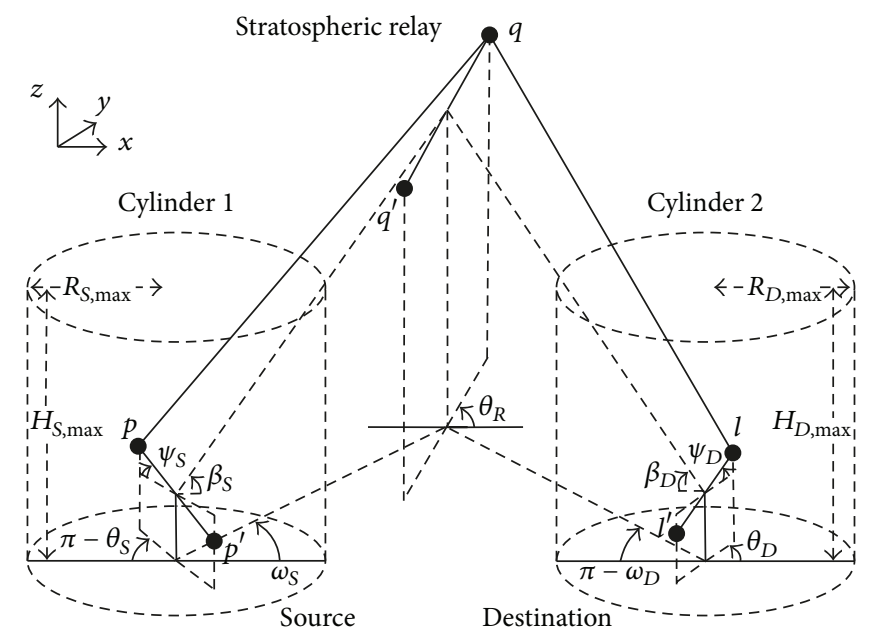

FIGURE 2: LoS paths of the 3D model for a $2 \times 2 \times 2$ MIMO MMSR channel.

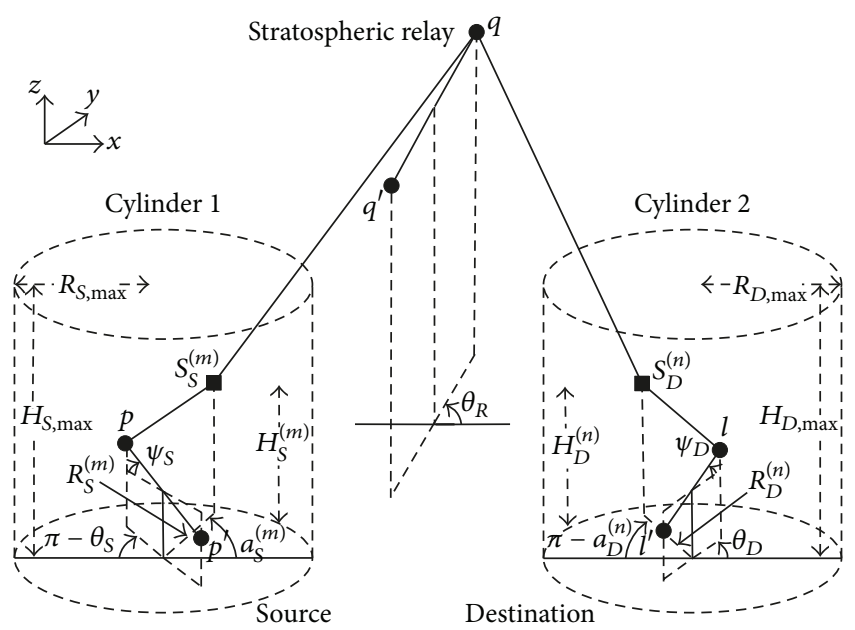

FIGURE 3: NLoS paths of the 3D model for a $2 \times 2 \times 2$ MIMO MMSR channel.

of $S_{S}^{(m)}\left(S_{D}^{(n)}\right)$ from the axis of the cylinder around the source (destination) is denoted by $R_{S}^{(m)}\left(R_{D}^{(n)}\right)$ and $H_{S}^{(m)}\left(H_{D}^{(n)}\right)$ stands for its height. Furthermore, $a_{S}^{(m)}$ is the azimuth angle of departure (AAoD) of the transmitted waves from the source to $S_{S}^{(m)}$ and $a_{D}^{(n)}$ is the azimuth angle of arrival (AAoA) of the waves scattered from $S_{D}^{(n)}$ and received at the destination. It is assumed that $a_{S}^{(m)}, a_{D}^{(n)}, R_{S}^{(m)}, R_{D}^{(n)}, H_{S}^{(m)}$, and $H_{D}^{(n)}$ are random variables and mutually independent.

The LoS component $h_{p q}^{S R, \mathrm{LoS}}=\left[\mathbf{H}_{S R, \mathrm{LoS}}\right]_{q p}$ of the impulse response for the transmission link from the pth source antenna element to the qth relay antenna element $(S-R$ subsystem) can be approximated as [23]

$$
h_{p q}^{S R-\operatorname{LoS}}=\zeta_{S R} b_{S}^{(p)} b_{S R}^{(q)},
$$

where

$$
\begin{gathered}
\zeta_{S R}=\exp \left(-j \frac{2 \pi}{\lambda} \frac{h_{R}}{\sin \beta_{S}}\right), \\
b_{S}^{(p)}=\exp \left[j \frac{(3-2 p) \pi \delta_{S} \cos \psi_{S} \cos \left(\theta_{S}-\omega_{S}\right)}{\lambda \cos \beta_{S}}\right], \\
b_{S R}^{(q)}=\exp \left[-j \frac{(3-2 q) \pi \delta_{R} \cos \left(\theta_{R}-\omega_{S}\right)}{\lambda \cos \beta_{S}}\right],
\end{gathered}
$$

$j^{2}=-1$, and $\lambda$ is the carrier wavelength. Then, using (8)-(11), the deterministic elements of $\mathbf{H}_{S R, \operatorname{LoS}}$ can be obtained as

$$
\mathbf{H}_{S R, \operatorname{LoS}}=\left[\begin{array}{ccc}
h_{11}^{S R-\operatorname{LoS}} & \ldots & h_{1 L_{T}}^{S R-\operatorname{LoS}} \\
\vdots & \ddots & \vdots \\
h_{L_{R} 1}^{S R-\operatorname{LoS} S} & \cdots & h_{L_{R} L_{T}}^{S R-\operatorname{LoS}}
\end{array}\right] .
$$

The MIMO channel matrix $\mathbf{H}_{R D \text {, LoS }}$ can be defined in a similar way as in $\mathbf{H}_{S R, \text { LoS }}$ by replacing the index $S$ by $D$.

The reference model assumes that the number of scatterers within cylinder 1 is infinite. Thus, the discrete random variables $a_{S}^{(m)}, R_{S}^{(m)}$, and $H_{S}^{(m)}$ can be replaced with continuous random variables $a_{S}, R_{S}$, and $H_{S}$ with joint probability density function (pdf) $f\left(a_{S}, R_{S}, H_{S}\right)$. Since $a_{S}^{(m)}, R_{S}^{(m)}$, and $H_{S}^{(m)}$ are independent, the joint pdf $f\left(a_{S}, R_{S}, H_{S}\right)$ can be decomposed to the product $f\left(a_{S}\right) f\left(R_{S}\right) f\left(H_{S}\right)$.

The von Mises pdf [26] (also known as the circular normal distribution) is used to characterize the $\mathrm{AAoD} a_{S}$ and is defined as

$$
f\left(a_{S}\right)=\frac{\exp \left[k_{S} \cos \left(a_{S}-\mu_{S}\right)\right]}{2 \pi I_{0}\left(k_{S}\right)}, \quad-\pi \leq a_{S} \leq \pi,
$$

where $I_{0}(\cdot)$ is the zeroth-order modified Bessel function of the first kind, $\mu_{S}$ is the mean angle at which the scatterers within cylinder 1 are distributed in the $x-y$ plane, and $k_{S} \geq 0$ controls the spread around the mean. This pdf is empirically justified in urban and suburban areas in [27]. Based on the experimental results in $[28,29]$, the hyperbolic pdf [30] is used to characterize $R_{S}$ and is defined as

$$
f\left(R_{S}\right)=\frac{u_{S}}{\tanh \left(u_{S} R_{S, \text { max }}\right) \cosh ^{2}\left(u_{S} R_{S}\right)}, \quad 0<R_{S} \leq R_{S, \text { max }} .
$$

The parameter $u_{S}$ controls the spread (standard deviation) of the scatterers around the source and its applicable value is in the interval $(0,1)$. Decreasing $u_{S}$ increases the spread of the pdf of $R_{S}$. Finally, the log-normal pdf [31] is used to characterize $H_{S}$ and is defined as

$$
f\left(H_{S}\right)=\frac{\exp \left[-\left[\ln \left(H_{S}\right)-\ln \left(H_{S, \text { mean }}\right)\right]^{2} /\left(2 \sigma_{S}^{2}\right)\right]}{H_{S} \sigma_{S} \sqrt{2 \pi}},
$$

where the parameters $H_{S, \text { mean }}$ and $\sigma_{S}$ are the mean and standard deviation of $H_{S}$. This pdf matched geographical data values obtained from measurements of building heights conducted in different areas [32,33]. 
Using (13)-(15) and after extensive calculation, the normalized NLoS component of the spatial correlation function (SCF) of the $S$ - $R$ subsystem is approximated as follows [23]:

$$
\begin{aligned}
& R_{p q, p^{\prime} q^{\prime}}^{S R-\mathrm{NLS}}\left(\delta_{S}, \delta_{R}\right) \\
& =\frac{\mathrm{E}\left[h_{p q}^{S R-\mathrm{NLoS}} h_{p^{\prime} q^{\prime}}^{S R-\mathrm{NLoS} S^{*}}\right]}{\sqrt{E\left[\left|h_{p q}^{S R-\mathrm{NLoS}}\right|^{2}\right] E\left[\mid h_{p^{\prime} q^{\prime}}^{\left.S R-\left.\mathrm{NLoS}\right|^{2}\right]}\right.}} \\
& =p_{S R, 1} \int_{0}^{H_{S, \max }} \int_{0}^{R_{S, \max }} p_{S R, 2} I_{0}\left(\sqrt{p_{S R, 3}^{2}+p_{S R, 4}^{2}}\right) d R_{S} d H_{S},
\end{aligned}
$$

where $h_{p q}^{S R \text {-NLoS }}$ is the NLoS component of the impulse response for the transmission link from the pth source antenna element to the $q$ th relay antenna element $(S-R$ MIMO subsystem), $(\cdot)^{*}$ denotes complex conjugate operation,

$$
\begin{gathered}
p_{S R, 1}=\frac{u_{S} b_{S R}^{(q)} b_{S R}^{\left(q^{\prime}\right)^{*}}}{\sigma_{S} \sqrt{2 \pi} \tanh \left(u_{S} R_{S, \max }\right) I_{0}\left(k_{S}\right)}, \\
p_{S R, 2}=\frac{\exp \left[-\left[\ln \left(H_{S}\right)-\ln \left(H_{S, \text { mean }}\right)\right]^{2} /\left(2 \sigma_{S}^{2}\right)\right]}{H_{S} \cosh ^{2}\left(u_{S} R_{S}\right)} \\
\times \exp \left\{j \frac{2 \pi}{\lambda}\left(p^{\prime}-p\right) \delta_{S} \sin \psi_{S}\right. \\
\left.\times \sin \left[\arctan \left(\frac{H_{S}}{R_{S}}\right)\right]\right\} \\
p_{S R, 3}=j \frac{2 \pi}{\lambda}\left\{\left(p^{\prime}-p\right) \delta_{S} \sin \theta_{S} \cos \psi_{S}\right. \\
+j \frac{2 \pi}{\lambda}\left[\frac{\left(q^{\prime}-q\right) \delta_{R} R_{S} \sin \beta_{S} \cos \omega_{S} \sin \left(\theta_{R}-\omega_{S}\right)}{h_{R} \cos \beta_{S}}\right] \\
\left.\left.\left.+k_{S} \sin \mu_{S}, \quad \frac{H_{S}}{R_{S}}\right)\right]\right\} \\
p_{S R, 4}=j \frac{2 \pi}{\lambda}\left\{\left(p^{\prime}-p\right) \delta_{S} \cos \theta_{S} \cos \psi_{S}\right. \\
+j \frac{2 \pi}{\lambda}\left[\frac{\left(q^{\prime}-q\right) \delta_{R} R_{S} \sin \beta_{S} \sin \omega_{S} \sin \left(\theta_{R}-\omega_{S}\right)}{h_{R} \cos \beta_{S}}\right] \\
\left.\times \cos \left[\arctan \left(\frac{H_{S}}{R_{S}}\right)\right]\right\}
\end{gathered}
$$

The double integral in (16) has to be numerically evaluated, since there is no closed-form solution. The elements of correlation matrix $\mathbf{R}_{S R, \mathrm{NLoS}}$ associated with the NLoS component of the $S$ - $R$ MIMO subsystem can be obtained using (16)-(20) as

$$
\mathbf{R}_{S R, N L o S}=\left[\begin{array}{cccc}
R_{11,11}^{S R \text {-NLoS }} & R_{11,21}^{S R \text {-NLoS }} & \cdots & R_{11, L_{R} L_{T}}^{S R \text {-NLoS }} \\
R_{21,11}^{S R \text {-NLS }} & \ddots & & R_{21, L_{R} L_{T}}^{S R \text {-NLS }} \\
\vdots & & \ddots & \vdots \\
R_{L_{R} L_{T}, 11}^{S R-N L S} & \cdots & & R_{L_{R} L_{T}, L_{R} L_{T}}^{S R \text { - LoS }}
\end{array}\right] .
$$

Since the number of scatterers within cylinder 2 is also infinite, the correlation matrix $\mathbf{R}_{R D, N L o s}$ associated with the NLoS component of the R-D MIMO can be derived by following a similar procedure to that used to derive $\mathbf{R}_{S R, N L o S}$.

\section{Results and Discussion}

In this section, the previously described theoretical derivations are demonstrated and the performance of a MIMO MMSR network over spatially correlated fading channels and MMSE receivers is studied in terms of BER for different system parameters and channel conditions. Unless indicated otherwise, the values of the model parameters used are depicted in Table 1. Note that we consider a typical densely built-up district (London [31]) to be the scattering region, that is, the surrounding buildings act as scatterers, and we set $H_{S \text {, mean }}=H_{D \text {,mean }}=17.6 \mathrm{~m}$ and $\sigma_{S}=\sigma_{D}=$ 0.31 . We also assume that $u_{S}=u_{D}=0.01$. As shown in Figure 4, this value corresponds to a reasonable average distance between the source (destination) and an effective scatterer of approximately $60 \mathrm{~m}$. The uplink ( $S$ - $R$ link) and the downlink ( $R-D$ link) may experience either symmetric, that is, double-Rayleigh (Rayleigh/Rayleigh) or double-Rician (Rician/Rician), or asymmetric [34], that is, Rayleigh/Rician or Rician/Rayleigh, fading phenomena depending on the strength of the LoS component. To clearly present the influence of the spatial correlation on the BER, we set the Rician factor of the $S-R$ and $R-D$ subsystems equal to zero, that is, $K_{S R}=K_{S R}=0$ (double-Rayleigh channel). Each transmitted frame is hierarchically modulated using QPSK for the high-priority (HP) stream and 16QAM for the lowpriority (LP) stream. Furthermore, each frame is further divided into 100 symbols, which are 4/16 hierarchically modulated and the channel is considered static during one frame period. Receivers with good reception conditions can decode successfully all 4 bits of each symbol, while receivers with bad conditions can decode only the high priority stream, which consists of the two first bits of each symbol.

The degree of spatial correlation is a complicated function of the degree of scattering in a specific propagation environment and the antenna inter-element spacing at the source, relay, and destination. Figure 5 examines the effect of the Rician factor on the BER performance. One observes that by increasing the Rician factors $K_{S R}\left(S-R\right.$ subsystem) and $K_{R D}$ $(R-D$ subsystem), there is a substantial degradation in BER for both streams. This is explained by the high correlation 
TABLE 1: System parameters used in the performance evaluation.

\begin{tabular}{|c|c|}
\hline Wireless network & Two-hop AF MMSR \\
\hline Transmission mode & $\begin{array}{l}\text { MIMO with two antennas at each node } \\
\qquad L_{S}=L_{R}=L_{D}=2\end{array}$ \\
\hline Frame size $(N)$ & 100 symbols \\
\hline Modulation & Hierarchical 4/16QAM \\
\hline Channel model & 3D geometry-based MIMO MMSR [23] \\
\hline Channel conditions & Static for one frame period \\
\hline Operating carrier frequency & $f=2 \mathrm{GHz}$ \\
\hline Carrier wavelength & $\lambda=0.15 \mathrm{~m}$ \\
\hline Rician factor of the S-R and R-D subsystems, respectively & $K_{\mathrm{SR}}=0, K_{\mathrm{RD}}=0$ (Double-Rayleigh $)$ \\
\hline Height of the relay antennas & $h_{R}=20 \mathrm{~km}$ \\
\hline $\begin{array}{l}\text { Azimuth angle of the relay with respect to the source and destination, } \\
\text { respectively }\end{array}$ & $\omega_{S}=\pi / 4, \omega_{D}=3 \pi / 4$ \\
\hline $\begin{array}{l}\text { Elevation angle of the relay with respect to the source and destination, } \\
\text { respectively }\end{array}$ & $\beta_{S}=\pi / 3, \beta_{D}=\pi / 3$ \\
\hline Antenna spacing at the source, relay, and destination, respectively & $\delta_{S}=\lambda, \delta_{R}=200 \lambda, \delta_{D}=\lambda$ \\
\hline $\begin{array}{l}\text { Orientation of antenna arrays at the source, relay, and destination, } \\
\text { respectively }\end{array}$ & $\theta_{S}=\pi / 2, \theta_{R}=\pi / 2, \theta_{D}=\pi / 2$ \\
\hline $\begin{array}{l}\text { Elevation angle of the } p \text { th source and the } l \text { th destination antenna } \\
\text { element, respectively }\end{array}$ & $\psi_{S}=\pi / 18, \psi_{D}=\pi / 18$ \\
\hline The radius of cylinders 1 and 2 , respectively & $R_{S, \max }=180 \mathrm{~m}, R_{D, \max }=180 \mathrm{~m}$ \\
\hline The height of cylinders 1 and 2 , respectively & $H_{S, \max }=70 \mathrm{~m}, H_{D, \max }=70 \mathrm{~m}$ \\
\hline $\begin{array}{l}\text { The spread of the scatterers within cylinders } 1 \text { and } 2 \text {, respectively, } \\
\text { around the mean azimuth angle }\end{array}$ & $k_{S}=5, k_{D}=5$ \\
\hline $\begin{array}{l}\text { Mean azimuth angle at which the scatterers are distributed within } \\
\text { cylinders } 1 \text { and } 2 \text {, respectively }\end{array}$ & $\mu_{S}=\pi / 6, \mu_{D}=\pi / 6$ \\
\hline $\begin{array}{l}\text { The spread of scatterers around the source and destination, } \\
\text { respectively }\end{array}$ & $u_{S}=0.01, u_{D}=0.01$ \\
\hline Mean height of the scatterers within cylinders 1 and 2, respectively & $H_{S \text {,mean }}=17.6 \mathrm{~m}, H_{D \text {,mean }}=17.6 \mathrm{~m}$ \\
\hline $\begin{array}{l}\text { Standard deviation of scatterers height within cylinders } 1 \text { and } 2 \text {, } \\
\text { respectively }\end{array}$ & $\sigma_{S}=0.31, \sigma_{D}=0.31$ \\
\hline
\end{tabular}

of the MIMO channels due to the increased strength of the LoS components. As the spatial correlation increases, an SNR loss is induced [35] and the BER deteriorates due to the difficulties experienced by the MMSE receiver, which tries to successfully separate the incoming streams. Hence, switching to single-input single-output (SISO) mode is suggested in this case. On the other hand, double-Rayleigh fading offers the required rich scattering environment that benefits MIMO transmission and then the streams are ideally decoded at the MMSE receiver. Moreover, a slight difference on the performance in the asymmetric cases exists, where the Rician/Rayleigh fading offers better results. This finding is further supported by the work in [34], which studied asymmetric fading conditions for an SISO system. Since AF relaying is considered in this cooperative mode, the channel conditions are mainly dominated by the first hop. Likewise, in our system we employ AF relaying and the signal reaches the relay with higher power in the Rician/Rayleigh case. Afterwards, in the $R-D$ link prior to detection, the signal experiences richer scattering which leads in less decoding errors compared to the Rayleigh/Rician case.
In Figure 6, the BER performance versus increasing values of antenna spacing at the source and destination is depicted for a fixed SNR of $20 \mathrm{~dB}$. The values of the antenna spacing are ranging from $\lambda / 4$ to $2 \lambda$. For this variation of the antenna spacing the spatial correlation remains low and amounts to nearly uncorrelated links. Therefore, the BER is not significantly affected for both streams.

Figure 7 investigates the effect of antenna spacing at the HAP, that is, $10 \lambda, 50 \lambda, 100 \lambda$, and $200 \lambda$, on the BER of the network for fixed SNR of $20 \mathrm{~dB}$. The results for both streams are depicted after the MMSE detection and decoding. These results indicate less channel correlation for increasing antenna spacing and this dramatically reflects on the BER of the network.

Figure 8 illustrates the BER performance for increasing values of the parameters $k_{S}$ (cylinder 1 ) and $k_{D}$ (cylinder 2) of the von Mises distribution in (13) for a fixed SNR of $20 \mathrm{~dB}$. The performance of the network does not experience an important degradation as the degree of scattering increases from 0 (isotropic scattering) to 10 and the MMSE receiver has almost stable performance. This increase has an analogous effect on channel correlation [36], which slightly increases. 


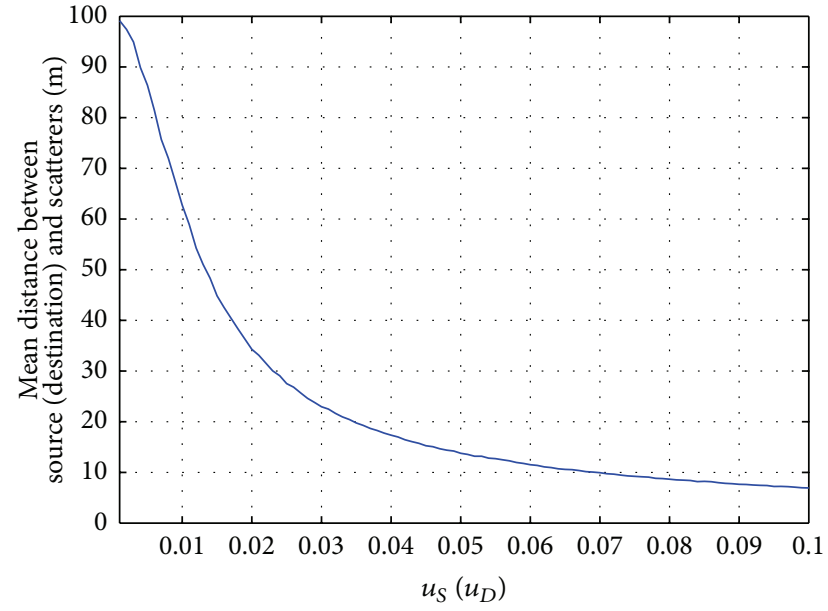

FIGURE 4: The mean distance between the source (destination) and the scatterers within cylinder 1 (2) for different values of the parameter $u_{S}\left(u_{D}\right)$.

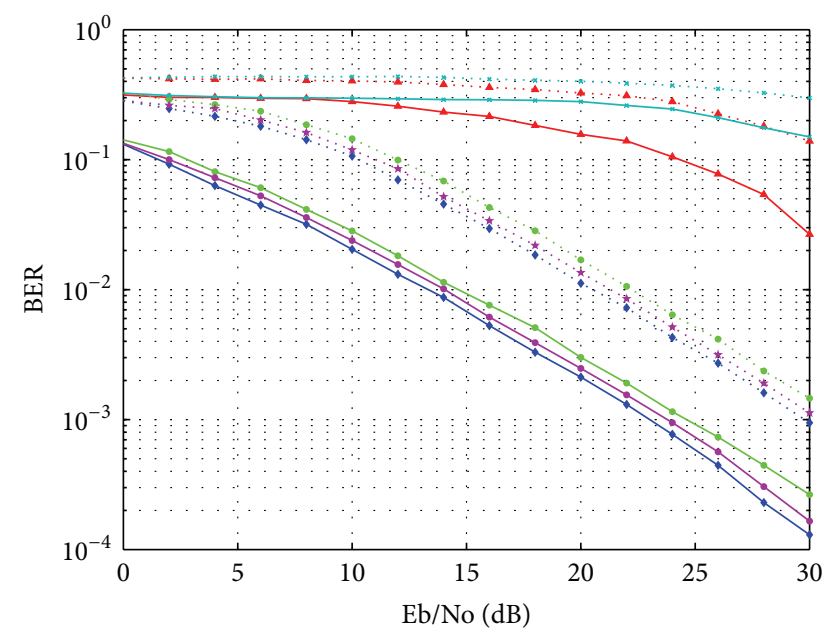

- HP double-Rayleigh $\left(K_{S R}=K_{R D}=0\right)$

$\cdots$ LP double-Rayleigh $\left(K_{S R}=K_{R D}=0\right)$

.... LP double-Rician $\left(K_{S R}=K_{R D}=1 \mathrm{~dB}\right)$

$\leadsto$ HP double-Rician $\left(K_{S R}=K_{R D}=1 \mathrm{~dB}\right)$

... LP Rayleigh-Rician $\left(K_{S R}=0, K_{R D}=1 \mathrm{~dB}\right)$

$\rightarrow$ HP Rayleigh-Rician $\left(K_{S R}=0, K_{R D}=1 \mathrm{~dB}\right)$

.*.. LP Rician-Rayleigh $\left(K_{S R}=1 \mathrm{~dB}, K_{R D}=0\right)$

$\rightarrow$ HP Rician-Rayleigh $\left(K_{S R}=1 \mathrm{~dB}, K_{R D}=0\right)$

…. LP double-Rician $\left(K_{S R}=K_{R D}=5 \mathrm{~dB}\right)$

$\longrightarrow$ HP double-Rician $\left(K_{S R}=K_{R D}=5 \mathrm{~dB}\right)$

FIGURE 5: BER for increasing the strength of the LoS component at the $S-R$ and $R-D$ subsystems.

Figure 9 illustrates the BER for a fixed SNR of $20 \mathrm{~dB}$ and for different spread of the scatterers in the vicinity of the source and destination, which is directly related with the hyperbolic distribution in (14). As the parameters $u_{S}$ (cylinder 1) and $u_{D}$ (cylinder 2) increase, the spread value decreases, and the correlation drastically increases [36]. Thus, the incoming streams are suboptimally demodulated at the

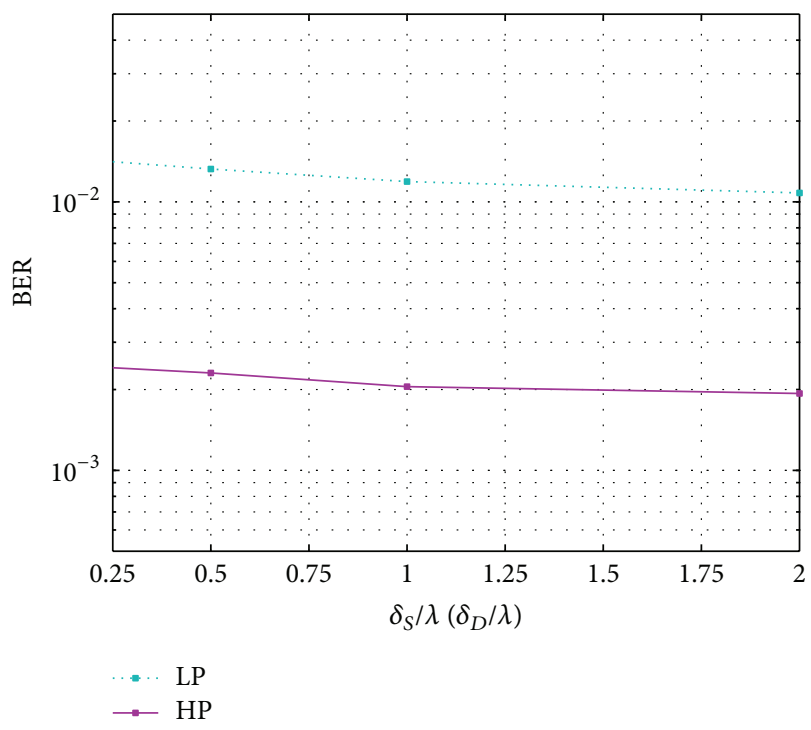

FIGURE 6: BER for increasing values of antenna inter-element spacing at the source and destination.

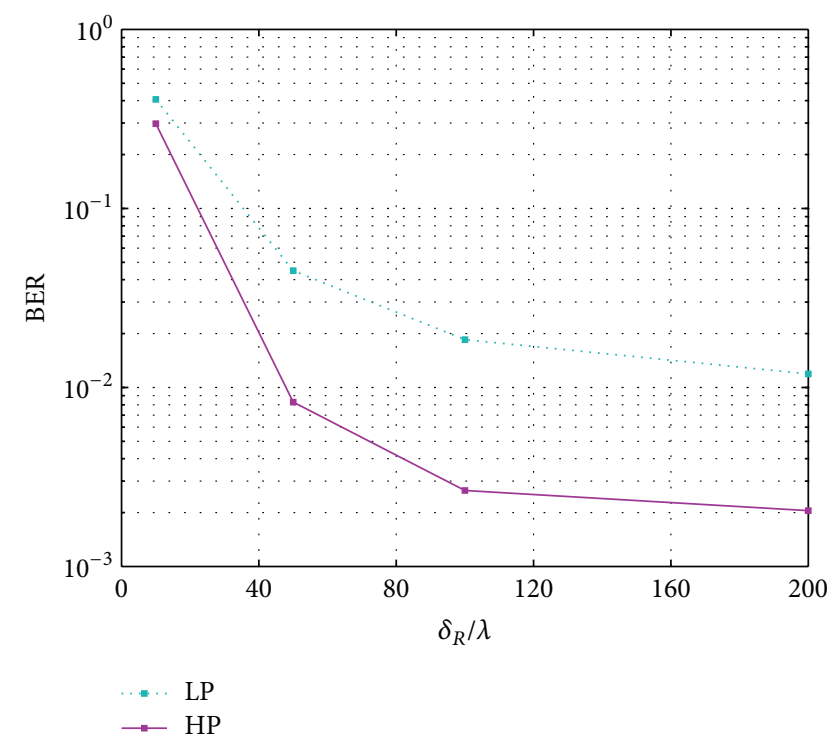

FIGURE 7: BER for increasing values of the antenna spacing at the stratospheric relay.

MMSE receiver and the BER performance is significantly degraded.

In Figure 10, different values for the mean height of the scatterers located within cylinders 1 and 2, that is, different $H_{S \text {,mean }}$ and $H_{D \text {,mean }}$, respectively, are considered (log-normal distribution), while the elevation angle of the source and destination antennas takes two different values: first, the elevation angles of the $p$ th source and the $l$ th destination antenna element are $\psi_{S}=\psi_{D}=\pi / 2$ and second, these angles are $\psi_{S}=\psi_{D}=\pi / 18$. As a general remark, increasing scatterers' mean height values, that is, increasing the degree of urbanization, leads to an insignificant decrease in the spatial correlation, which in turn explains the minor increase in the 


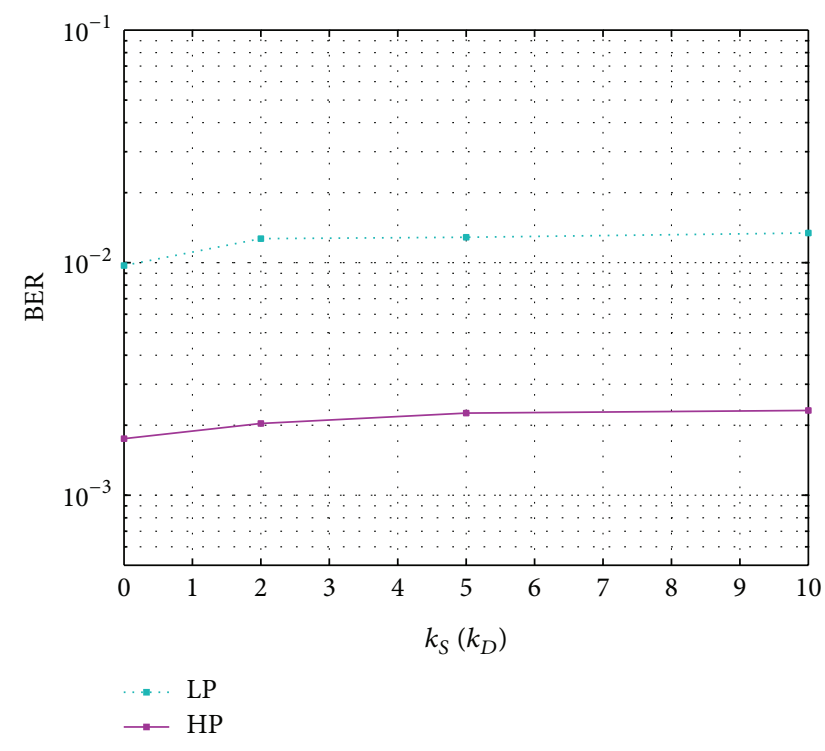

FIGURE 8: BER for increasing values of the degree of scattering within cylinders 1 and 2.

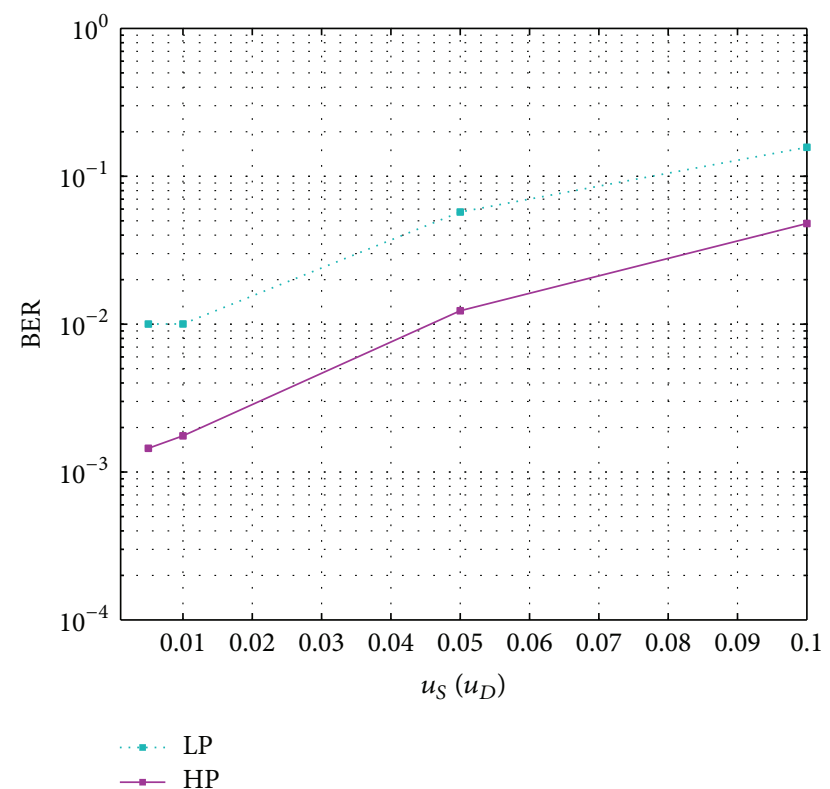

FIGURE 9: BER for increasing values of the average spread of scatterers around the source and destination.

BER performance for a fixed SNR of $20 \mathrm{~dB}$. Furthermore, for $\psi_{S}=\psi_{D}=\pi / 18$ better channel conditions are obtained, as indicated by the BER curves. Overall, Figure 10 asseverates the inclusion of the third dimension of the model in [23].

\section{Conclusions and Future Research Directions}

HAPs could be used to relay information between a source and one or many destinations providing the means for

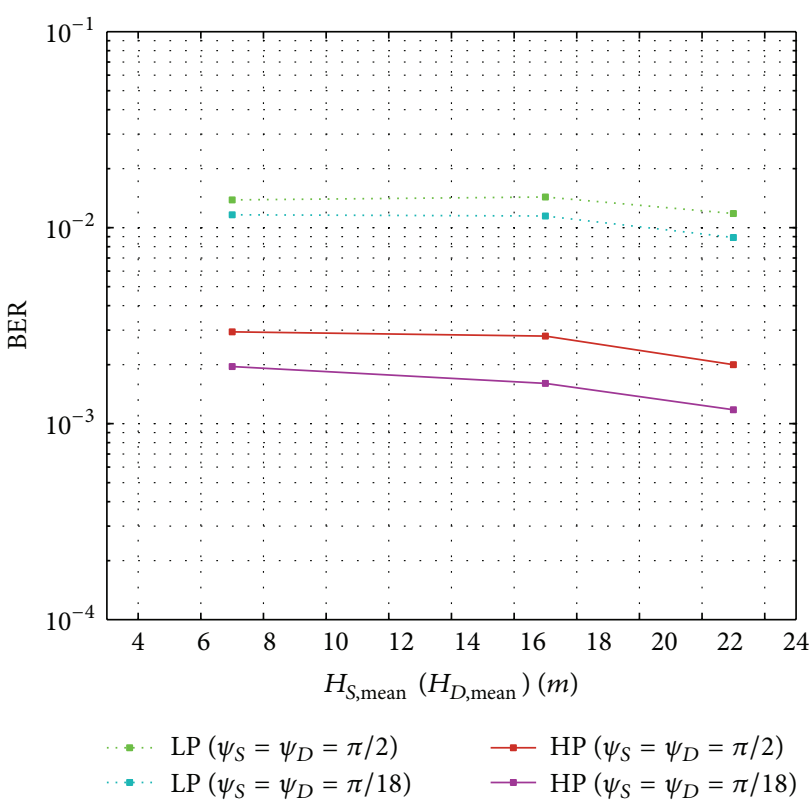

FIGURE 10: BER for increasing values of the mean height of the scatterers within cylinders 1 and 2.

integrated service provision in conjunction with terrestrial networks. Therefore, this paper has analyzed the BER performance of MIMO MMSR systems employing MMSE receivers in spatially correlated double-Rician fading channels. Through rigorous simulations, the BER of the hierarchically modulated transmitted symbols has been investigated in various scenarios. Results have revealed the relationship between channel correlation and BER for varying fading condition and distribution of the scatterers, thus illustrating valuable information for a stratospheric relay system implementation.

Future directions include the use of the 3D channel model in more complex scenarios, where integrated satellite-HAPterrestrial architectures are considered. Also, other relaying protocols such as decode-and-forward (DF) could be investigated in order to examine the effect of channel correlation in this type of operation. Finally, employing opportunistic relaying either in HAP selection or in relay selection on the ground is an attracting research field and the use of the $3 \mathrm{D}$ scattering model could provide interesting results depending on channel correlation conditions.

\section{Acknowledgments}

This work has been co-financed by the European Union (European Social Fund-ESF) and Greek national funds through the Operational Program "Education and Lifelong Learning" of the National Strategic Reference Framework (NSRF)-Research Funding Program THALES MIMOSA (MIS: 380041). Investing in knowledge society through the European Social Fund. 


\section{References}

[1] A. Nosratinia, T. E. Hunter, and A. Hedayat, "Cooperative communication in wireless networks," IEEE Communications Magazine, vol. 42, no. 10, pp. 74-80, 2004.

[2] J. N. Laneman, D. N. C. Tse, and G. W. Wornell, "Cooperative diversity in wireless networks: efficient protocols and outage behavior," IEEE Transactions on Information Theory, vol. 50, no. 12, pp. 3062-3080, 2004.

[3] B. Paillassa, B. Escrig, R. Dhaou, M. Boucheret, and C. Bes, "Improving satellite services with cooperative communications," International Journal of Satellite Communications and Networking, vol. 29, no. 6, pp. 479-500, 2011.

[4] B. Evans, M. Werner, E. Lutz et al., "Integration of satellite and terrestrial systems in future multimedia communications," IEEE Wireless Communications, vol. 12, no. 5, pp. 72-80, 2005.

[5] R. Giuliano, M. Luglio, and F. Mazzenga, "Interoperability between WiMAX and broadband mobile space networks," IEEE Communications Magazine, vol. 46, no. 3, pp. 50-57, 2008.

[6] S. Kota, G. Giambene, and S. Kim, "Satellite component of NGN: integrated and hybrid networks," International Journal of Satellite Communications and Networking, vol. 29, no. 3, pp. 191208, 2011.

[7] A. Mohammed, A. Mehmood, F. Pavlidou, and M. Mohorcic, "The role of high-altitude platforms (HAPs) in the global wireless connectivity," Proceedings of the IEEE, vol. 99, no. 11, pp. 1939-1953, 2011.

[8] D. Avagnina, F. Dovis, A. Ghiglione, and P. Mulassano, "Wireless networks based on high-altitude platforms for the provision of integrated navigation/communication services," IEEE Communications Magazine, vol. 40, no. 2, pp. 119-125, 2002.

[9] E. Falletti, M. Laddomada, M. Mondin, and F. Sellone, "Integrated services from high-altitude platforms: a flexible communication system," IEEE Communications Magazine, vol. 44, no. 2, pp. 85-94, 2006.

[10] M. Antonini, E. Cianca, A. De Luise, M. Pratesi, and M. Ruggieri, "Stratospheric relay: potentialities of new satellitehigh altitude platforms integrated scenarios," in Proceedings of the IEEE Aerospace Conference, vol. 3, pp. 3-1211-3-1219, March 2003.

[11] G. W. Jull, A. Lillemark, and R. M. Turner, "SHARP (stationary high altitude relay platform) telecommunications missions and systems," in Proceedings of the IEEE Global Telecommunications Conference, pp. 955-959, New Orleans, La, USA, December 1985.

[12] G. M. Djuknic, J. Freidenfelds, and Y. Okunev, "Establishing wireless communications services via high-altitude aeronautical platforms: a concept whose time has come?" IEEE Communications Magazine, vol. 35, no. 9, pp. 128-135, 1997.

[13] M. Oodo, H. Tsuji, R. Miura et al., "Experiments on IMT-2000 using unmanned solar powered aircraft at an altitude of $20 \mathrm{~km}$," IEEE Transactions on Vehicular Technology, vol. 54, no. 4, pp. 1278-1294, 2005.

[14] P. Zhan, K. Yu, and A. L. Swindlehurst, "Wireless relay communications with unmanned aerial vehicles: performance and optimization," IEEE Transactions on Aerospace and Electronic Systems, vol. 47, no. 3, pp. 2068-2085, 2011.

[15] A. Paulraj, R. Nabar, and D. Gore, Introduction to SpaceTime Wireless Communications, Cambridge University Press, Cambridge, UK, 2003.
[16] H. Muhaidat and M. Uysal, "Cooperative diversity with multiple-antenna nodes in fading relay channels," IEEE Transactions on Wireless Communications, vol. 7, no. 8, pp. 30363046, 2008.

[17] R. U. Nabar, H. Bölcskei, and A. J. Paulraj, "Diversity and outage performance in space-time block coded ricean MIMO channels," IEEE Transactions on Wireless Communications, vol. 4, no. 5, pp. 2519-2532, 2005.

[18] A. G. Armada, L. Hong, and A. Lozano, "Bit loading for MIMO with statistical channel information at the transmitter and ZF receivers," in Proceedings of the IEEE International Conference on Communications, pp. 1-5, Dresden, Germany, June 2009.

[19] R. W. Heath and D. J. Love, "Multimode antenna selection for spatial multiplexing systems with linear receivers," IEEE Transactions on Signal Processing, vol. 53, no. 8, pp. 3042-3056, 2005.

[20] J. G. Proakis, Digital Communications, McGraw-Hill, New York, NY, USA, 3rd edition, 1995.

[21] Y. Jiang, M. K. Varanasi, and J. Li, "Performance analysis of ZF and MMSE equalizers for MIMO systems: an in-depth study of the high SNR regime," IEEE Transactions on Information Theory, vol. 57, no. 4, pp. 2008-2026, 2011.

[22] A. Schertz and C. Week, "Hierarchical modulation-the transmission of two independent DVB-T multiplexes on a single frequency," EBU Technical Review, no. 294, 13 pages, 2003.

[23] E. T. Michailidis, P. Theofilakos, and A. G. Kanatas, “Threedimensional modeling and simulation of MIMO mobile-tomobile via stratospheric relay fading channels," IEEE Transactions on Vehicular Technology, no. 99, 1 pages, 2012.

[24] C. S. Patel, G. L. Stüber, and T. G. Pratt, "Statistical properties of amplify and forward relay fading channels," IEEE Transactions on Vehicular Technology, vol. 55, no. 1, pp. 1-9, 2006.

[25] D. Shiu, G. J. Foschini, M. J. Gans, and J. M. Kahn, "Fading correlation and its effect on the capacity of multielement antenna systems," IEEE Transactions on Communications, vol. 48, no. 3, pp. 502-513, 2000.

[26] A. Abdi and M. Kaveh, "A space-time correlation model for multielement antenna systems in mobile fading channels," IEEE Journal on Selected Areas in Communications, vol. 20, no. 3, pp. 550-560, 2002.

[27] A. Abdi, J. A. Barger, and M. Kaveh, "A parametric model for the distribution of the angle of arrival and the associated correlation function and power spectrum at the mobile station," IEEE Transactions on Vehicular Technology, vol. 51, no. 3, pp. 425-434, 2002.

[28] S. S. Mahmoud, Z. M. Hussain, and P. O'Shea, "A geometricalbased microcell mobile radio channel model," Wireless Networks, vol. 12, no. 5, pp. 653-664, 2006.

[29] S. S. Mahmoud, F. S. Al-Qahtani, Z. M. Hussain, and A. Gopalakrishnan, "Spatial and temporal statistics for the geometrical-based hyperbolic macrocell channel model," Digital Signal Processing, vol. 18, no. 2, pp. 151-167, 2008.

[30] S. S. Mahmoud, Z. M. Hussain, and P. O'Shea, "A space-time model for mobile radio channel with hyperbolically distributed scatterers," IEEE Antennas and Wireless Propagation Letters, vol. 1, pp. 211-214, 2002.

[31] M. Vazquez-Castro, F. Perez-Fontan, and S. R. Saunders, "Shadowing correlation assessment and modeling for satellite diversity in urban environments," International Journal of Satellite Communications, vol. 20, no. 2, pp. 151-166, 2002. 
[32] M. A. Vázquez-Castro, D. Belay-Zelek, and A. CuriesesGuerrero, "Availability of systems based on satellites with spatial diversity and HAPS," Electronics Letters, vol. 38, no. 6, pp. 286288, 2002.

[33] C. Tzaras, E. G. Evans, and S. R. Saunders, "Physical-statistical analysis of land mobilesatellite channel," Electronics Letters, vol. 34, no. 13, pp. 1355-1357, 1998.

[34] H. Suraweera, G. K. Karagiannidis, and P. J. Smith, "Performance analysis of the dual-hop asymmetric fading channel," IEEE Transactions on Wireless Communications, vol. 8, no. 6, pp. 2783-2788, 2009.

[35] M. R. McKay and I. B. Collings, "Error performance of MIMO-BICM with zero-forcing receivers in spatiallycorrelated Rayleigh channels," IEEE Transactions on Wireless Communications, vol. 6, no. 3, pp. 787-792, 2007.

[36] E. T. Michailidis and A. G. Kanatas, "Three-dimensional HAPMIMO channels: modeling and analysis of space-time correlation," IEEE Transactions on Vehicular Technology, vol. 59, no. 5, pp. 2232-2242, 2010. 

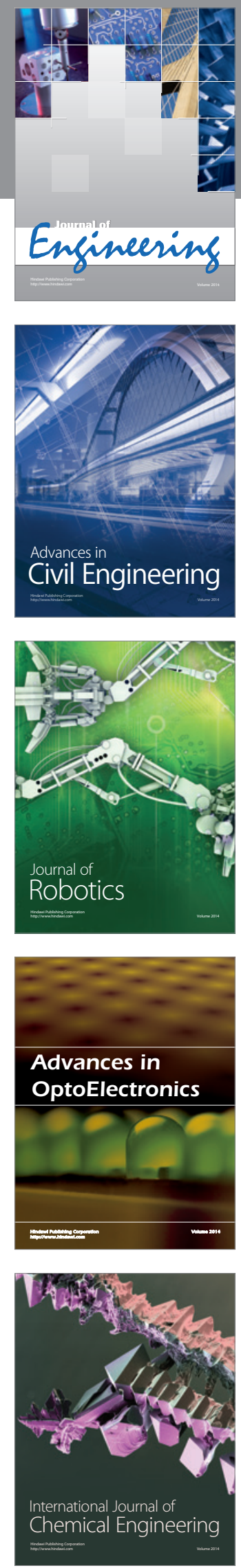

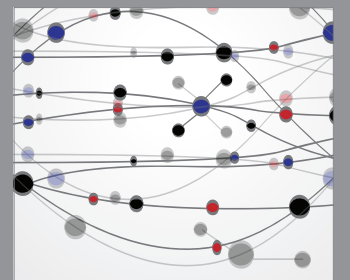

The Scientific World Journal
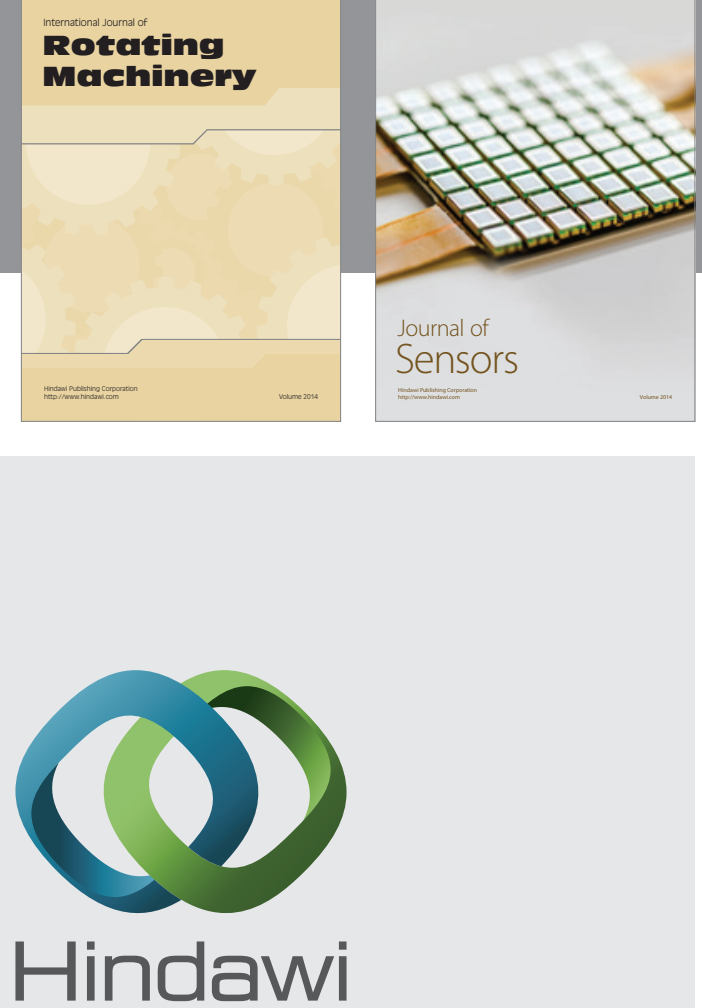

Submit your manuscripts at http://www.hindawi.com
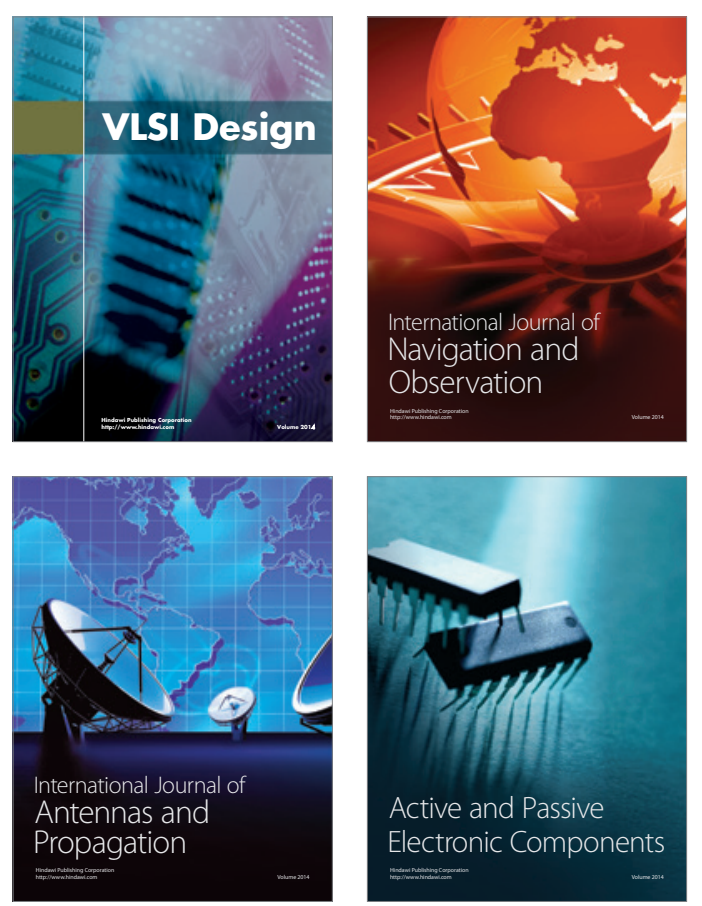
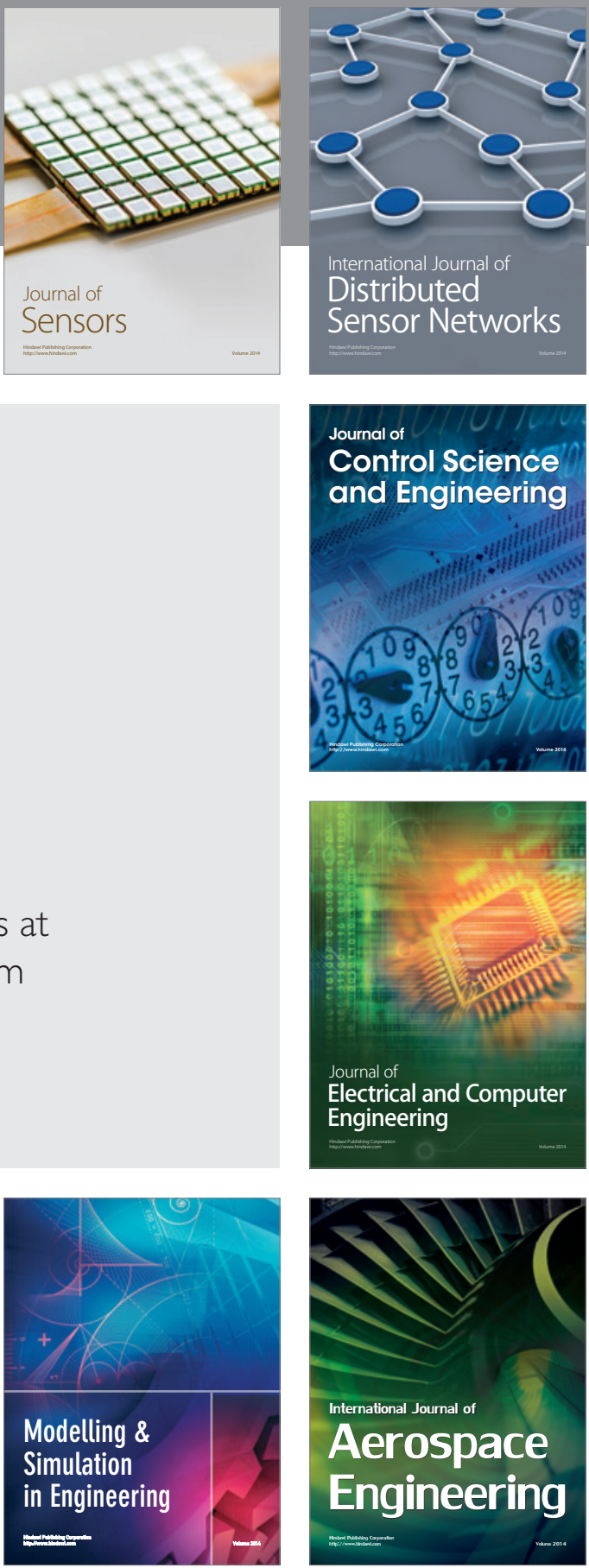

Journal of

Control Science

and Engineering
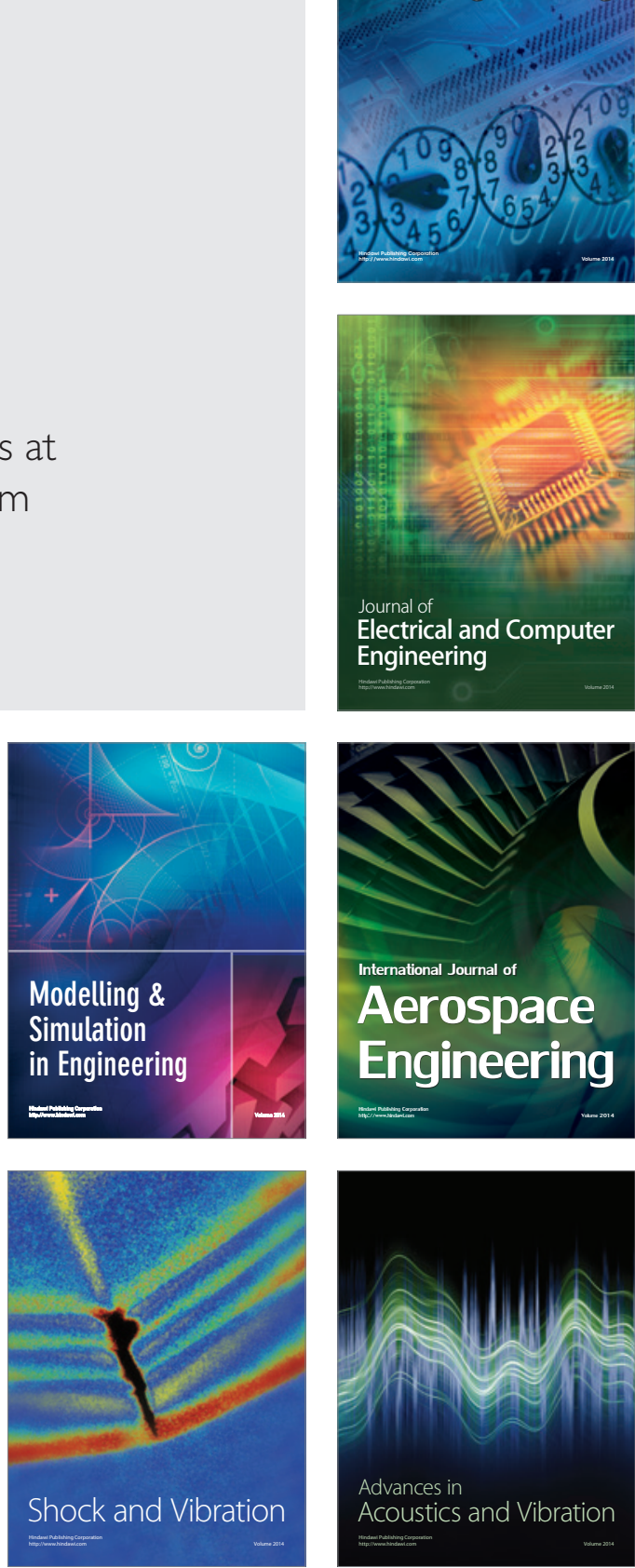